\title{
Security in Telemedicine using DWT-CDCS
}

\author{
Kulkarni Pratibha C. \\ Dept. of Electronics \& Telecommunication \\ JSPM's Imperial College of Engineering \& \\ Research, Wagholi, Pune, India
}

\author{
Bhatia S.K. \\ Dept. of Electronics\& Telecommunication \\ JSPM's Imperial College of Engineering \& Research \\ Wagholi, Pune, India
}

\begin{abstract}
Copyright protection of digital media is the very first application that comes to mind for digital watermarking. In the past, duplicating artwork was quite complicated and requires great efforts to create the work looks just like the original. However, in present digital world it is very simple for anyone to duplicate or manipulate digital data. The digital image watermarking allows the watermark to be embedded visibly or invisibly in the original image for identification of the owner. This concept can also be used for other media, such as digital video and audio.
\end{abstract}

Telemedicine is a well-known application of digital watermarking. In this application security \& authentication for medical data is very important. Hiding the data into the medical image provide the security over the public network. Authentication verifies whether the image certainly belongs to the right patient Authentication of medical data used for further diagnosis and reference.

This paper focused on the methods of medical image data hiding for security and authentication. High capacity data hiding is achieved with CDCS (Class Dependent Coding Scheme). As well as data is effectively hided with discrete wavelet transform.

Embedding watermarks in RONI (region of non interest) protects the ROI (region of interest) of medical image, which is diagnostically important part of medical images. Segmentation plays an important role in medical image processing for separating the ROI from medical image.

\section{General Terms}

Medical image segmentation is done to protect diagnostically important part called region of interest (ROI). Text embedding is done in the rest part of an image called region of non interest (RONI).

Also medical data can be embedded with LSB technique in medical image which is processed with discrete cosine transform

\section{Keywords}

CDCS, data hiding, medical image, ROI

\section{INTRODUCTION}

Now days, because of great development in technologies, the business related applications are running on digital way for example communication, networked multimedia system, and digital data storage etc. After the revolution in internet \& digital world all the business environment are achieving effectiveness \& security using digitalization of business application with the help of internet. Telemedicine is one of the important applications, where healthcare professional uses internet to transfer or receive the medical data[15].The biomedical images which can be readily shared by using computer networks and are easily available to use, also these can be processed as well as transmitted with internet.
Telemedicine reduces distance barrier due to that it is easy to provide medical services in remote areas which are not accessible easily. It is useful in critical and emergency situations of patients. Medical diagnosis determines a possible disorder or diseases. Diagnosis based on patient's consultation, physical examination and medical tests. Medical test such as laboratory, biosignal (ECG, EEG), Image analysis etc are used, shown in figure below.

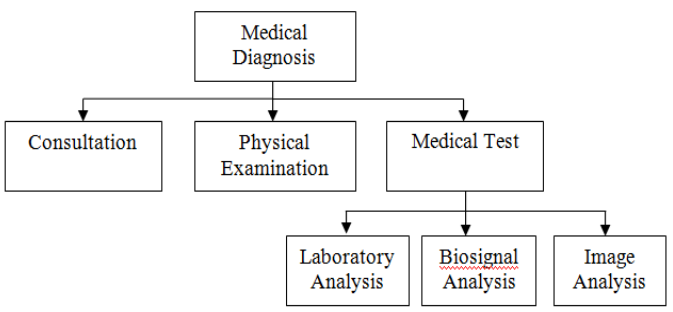

Figure 1 Medical Diagnoses

Figure 1 illustrate typical e-diagnosis model. Physician can get patient report via email or any web tool through the internet. Doctor can refer digital report of the patient to other experts to get the second opinion. For the future diagnosis, this medical data can be stored in the healthcare's database [3]. As the critical diagnosis are depends on medical images or medical data, security and confidentiality are the main issues in it [15]. So that medical data should not be changed by unauthorized person otherwise, patient suffers from wrong treatment due to the change in diagnosis information. That's why medical data should have highly secured. In recent years, due to wide use of internet, it is more convenient to transfer the medical images or patient information regarding his/her diagnosis among hospitals, which are located in the different geographical location. Many times this referenced medical data transfer is done via unsecured open networks. These will leads to change in medical images (for example, one patient's reports or medical images are replaced by other patient) are makes the wrong diagnosis. Considering this fact, medical data should have highly secured.

Embedding of the watermark into medical image is process for copyright protection $[3,8]$. And authentication of that medical image is achieved by extracting watermark. In embedding process, secrete data in the form of text, image or audio has been embedded into original medical image and resultant image is call watermarked image.

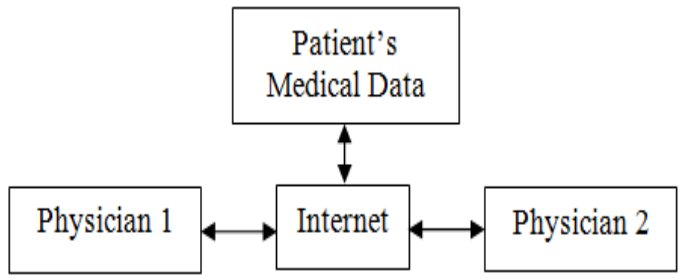

Figure 2 Typical e-diagnosis models 
In extraction processing, secret data is extracted from the watermarked image. If there is error in extraction process, it will show that image gets tampered. Physician does not diagnose on such tampered images.

\section{PROPOSED SYSTEM}

Proposed work describes an image watermarking method for medical images. This proposed method helps to increases the security and capacity of robust watermark. Patient's report is used as a watermark. To increase the capacity of hiding data, class dependent coding scheme is used. Discrete wavelet transform is used to decompose the cover image into four subbands each of same size as that of the original image. By embedding the watermark in the four subbands the proposed scheme became more robust to common attacks and achieves secure data transfer. Proposed work consist blind watermarking technique and tested the algorithm for various attacks. Blind watermarking is based on CDCS-DWT algorithm. Since high hiding capacity is possible with CDCS as well as it effectively embedded into medical image and scheme became more robust to common attacks hence the name High capacity \& effective medical image data hiding using CDCS.

\subsection{Watermark Embedding Algorithm}

1. Medical image $(\mathrm{N} \times \mathrm{N})$ acquisition \& convert into grayscale image of size $512 \times 512 \&$ is used as input image.

2. Input image separated into two parts - Region Of Interest (ROI) \& Region Of Non Interest (RONI) by using GUI

3. Data (Text file containing patient report) acquisition \& convert each character into 6 bit binary number by using CDCS

4. Redundant \& interleaving bits are added in CDCS code

5. Divide RONI image into $8 \times 8$ blocks

6. Apply DWT to each block of size $8 \times 8$, block decomposed into four subbands LL, LH, HL, HH.

7. Select valid blocks \& quantized, non-zero predefined coefficients are considered for embedding the data

8. By using LSB method CDCS bits are embedded into non-zero coefficients

9. Apply IDWT to subbands \& reconstruct block of size $8 \times 8$

10. Combine all blocks to form embedded RONI image

11. Watermarked image is obtained by combining embedded RONI \& ROI images.

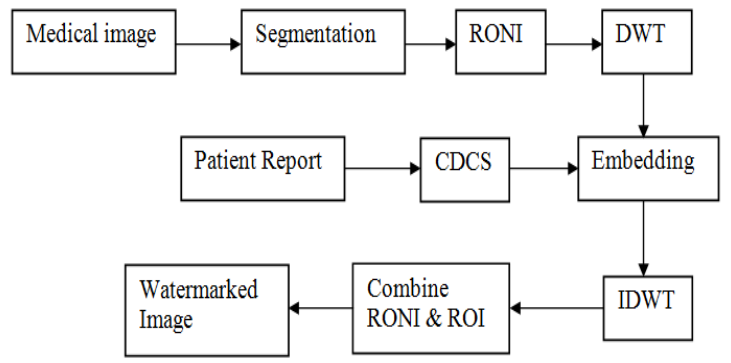

Figure 3 Block Diagram of Embedding Process

\subsection{Watermark Extraction Algorithm}

The watermark extraction process is similar to that of embedding one except that at the receiving end extractor should have the knowledge of location of the embedded watermark. This can achieve by the key-based embedding and detection. With this type of method access to the watermark by unauthorized users is prevented.

1. Watermarked image separated into two parts Region Of Interest (ROI) \& Region Of Non Interest (RONI) by using GUI

2. Divide RONI image into $8 \times 8$ blocks

3. Apply DWT to each block of size $8 \times 8$, block decomposed into four subbands LL, LH, HL, HH

4. Select valid blocks \& extract data bits from predefined coefficients

5. Apply Inverse CDCS to extracted bits \& reconstruct patient report

6. Calculate bit error rate \& correlation coefficient of ROI

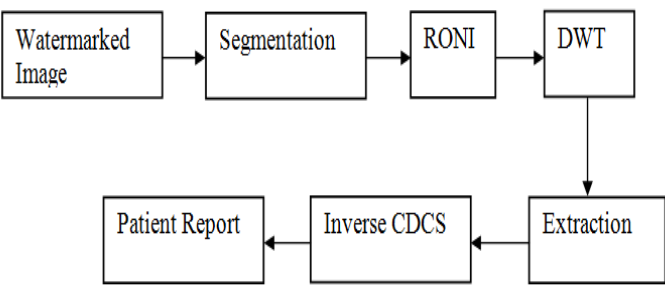

Figure 4 Block Diagram of Extraction Process

\section{METHODOLOGY}

\subsection{Class Dependent Coding Scheme}

Class dependent coding scheme is the simple technique which is based on probability of the occurrence of characters used in patient report [1]. Characters are divided into three categories, class A, class B, \& class C. Most frequently used characters are comes under Class A. Class B consists less or average frequently used characters and Class $\mathrm{C}$ consist rarely used characters. Each class has two bit code $00,01 \& 10$ for Class A, $\mathrm{B}, \& \mathrm{C}$ respectively.

Class dependent coding scheme consist capital letters, special and alphanumeric characters. Based on Huffman encoding, the variable length class code (CC) have been designed to represent each class.

\subsection{DWT}

The image is high and low-pass filtered along the rows and the results of each filter are down- sampled by two. Those two sub-signals correspond to the high and low frequency components along the rows and are each of size $\mathrm{N}$ by N/2. Each of those sub-signals is then again high and low-pass filtered, but this time along the column data. The results are again down-sampled by two. $\mathrm{h}(-\mathrm{m})$ and $\mathrm{g}(-\mathrm{m})$ are the low pass and high pass analysis filters, while the corresponding low pass and high pass synthesis filters are $\mathrm{h}(\mathrm{m})$ and $\mathrm{g}(\mathrm{m}) ; \mathrm{c}_{\mathrm{j}}$ and $\mathrm{d}_{\mathrm{j}}$ are the low band and high band output coefficients at level j.

DWT analysis is given by [29]

$$
\begin{aligned}
& c_{j+1}[m, n]=\left(c_{j}(m, n) * h[-m]\right) \downarrow 2 \\
& d_{j+1}[m, n]=\left(c_{j}(m, n) * g[-m]\right) \downarrow 2
\end{aligned}
$$

Similarly DWT synthesis is given by 


$$
\begin{gathered}
c_{j+1}[m, n]=\left[\left(c_{j}(m, n) \uparrow 2\right) * h[m]+\right. \\
\left.\left(d_{j}(m, n) \uparrow 2\right) * g[m]\right]
\end{gathered}
$$

\subsection{Robustness Test}

To ensure the reliability and quality of the watermarked image, the performance of watermarking is calculated, which measured in terms of perceptibility. There are two method of calculating the performance measure_ Mean Square Error (MSE) is simplest function to measure the perceptual distance between watermarked and original image.

$$
M S E=1 \div n \sum_{i}^{n}\left(I^{\prime}-I\right)^{2}
$$

Where, I is original image and I is watermarked image.

Peak Signal to Noise Ratio (PSNR) is used to measure the similarity between images before and after watermarking.

$P S N R=10 \log _{10} \max I^{2} \div M S E$

Where, max I is the peak value of original image.

\section{RESULT}

The proposed blind algorithm is tested with different medical images. These medical images are taken from eye hospital, dental hospital and medical case study websites.

In the proposed work, different Patient's reports with various data capacity are selected. Those reports are converted into the CDCS bits. Redundancy \& interleaving bits are added prior to each CDCS bit. Capacity of All this medical data has tested on DWT -CDCS algorithm. Salt \& paper noise is applied to check the PSNR value and BER.

Results are shown in table below for given medical image. Figure 5, $6 \& 7$ shows graphic representation of PSNR, MSE, BER and NCC in table 1.

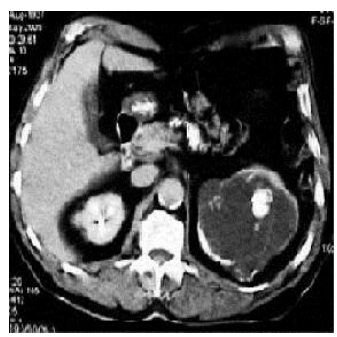

Image 1

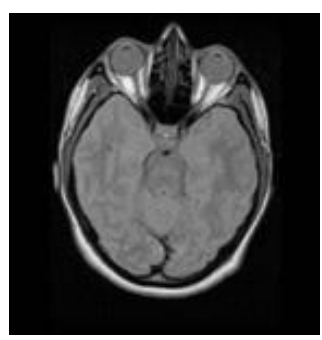

Image 2
Table 1 DWT-CDCS Result on Image 1

DWT-CDCS Results after various attacks on Image 1

\begin{tabular}{|c|c|c|c|c|c|}
\hline Attacks & $\begin{array}{c}\text { PBS } \\
(\%)\end{array}$ & MSE & $\begin{array}{c}\text { PSNR } \\
(\mathbf{d B})\end{array}$ & $\begin{array}{c}\text { BER } \\
(\%)\end{array}$ & $\begin{array}{c}\text { NCC } \\
\text { of } \\
\text { ROI }\end{array}$ \\
\hline $\begin{array}{c}\text { Without } \\
\text { Attack }\end{array}$ & $\begin{array}{c}29.166 \\
7\end{array}$ & $\begin{array}{c}0.3026 \\
5\end{array}$ & $\begin{array}{c}53.321 \\
4\end{array}$ & 0 & $\begin{array}{c}0.9883 \\
4\end{array}$ \\
\hline $\begin{array}{c}\text { Salt \& } \\
\text { Paper }\end{array}$ & $\begin{array}{c}29.166 \\
7\end{array}$ & $\begin{array}{c}0.5779 \\
1\end{array}$ & $\begin{array}{c}50.512 \\
2\end{array}$ & 6.2325 & $\begin{array}{c}0.9893 \\
7\end{array}$ \\
\hline Gaussian & $\begin{array}{c}29.166 \\
7\end{array}$ & $\begin{array}{c}16.413 \\
9\end{array}$ & $\begin{array}{c}35.978 \\
7\end{array}$ & 48.109 & $\begin{array}{c}0.9477 \\
1\end{array}$ \\
\hline Speckle & $\begin{array}{c}29.166 \\
7\end{array}$ & $\begin{array}{c}12.425 \\
1\end{array}$ & $\begin{array}{c}37.187 \\
8\end{array}$ & $\begin{array}{c}21.638 \\
7\end{array}$ & $\begin{array}{c}0.9405 \\
8\end{array}$ \\
\hline
\end{tabular}

\begin{tabular}{|c|c|c|c|c|c|} 
Poisson & $\begin{array}{c}29.166 \\
7\end{array}$ & 5.4603 & $\begin{array}{c}40.758 \\
7\end{array}$ & $\begin{array}{c}28.291 \\
3\end{array}$ & $\begin{array}{c}0.9568 \\
5\end{array}$ \\
\hline Blurring & $\begin{array}{c}29.166 \\
7\end{array}$ & 2.0662 & $\begin{array}{c}44.979 \\
1\end{array}$ & $\begin{array}{c}21.288 \\
5\end{array}$ & $\begin{array}{c}0.9852 \\
5\end{array}$ \\
\hline $\begin{array}{c}\text { Sharpenin } \\
\text { g }\end{array}$ & $\begin{array}{c}29.166 \\
7\end{array}$ & $\begin{array}{c}10.770 \\
6\end{array}$ & $\begin{array}{c}37.808 \\
4\end{array}$ & $\begin{array}{c}51.750 \\
7\end{array}$ & $\begin{array}{c}0.9667 \\
7\end{array}$ \\
\hline $\begin{array}{c}\text { Rotation } \\
\text { by } 90\end{array}$ & $\begin{array}{c}29.166 \\
7\end{array}$ & $\begin{array}{c}84.128 \\
4\end{array}$ & $\begin{array}{c}28.881 \\
4\end{array}$ & $\begin{array}{c}45.238 \\
1\end{array}$ & $\begin{array}{c}0.1385 \\
7\end{array}$ \\
\hline
\end{tabular}

Table 2 DWT-CDCS Result on Image 2

\begin{tabular}{|l|c|c|c|c|c|}
\hline \multicolumn{5}{|c|}{ DWT-CDCS Results after various attacks on Image 2 } \\
\hline Attacks & $\begin{array}{c}\text { PBS } \\
(\%)\end{array}$ & MSE & $\begin{array}{c}\text { PSNR } \\
(\mathbf{d B})\end{array}$ & $\begin{array}{c}\text { BER } \\
(\%)\end{array}$ & $\begin{array}{c}\text { NCC of } \\
\text { ROI }\end{array}$ \\
\hline $\begin{array}{l}\text { Without } \\
\text { Attack }\end{array}$ & 29.1667 & 0.27713 & 53.704 & 0 & 0.66269 \\
\hline $\begin{array}{l}\text { Salt \& } \\
\text { Paper }\end{array}$ & 29.1667 & 0.53577 & 50.841 & 1.4706 & 0.95483 \\
\hline Gaussian & 29.1667 & 15.3999 & 36.2556 & 51.4706 & 0.53685 \\
\hline Speckle & 29.1667 & 8.7255 & 38.7244 & 30.042 & 0.861 \\
\hline Poisson & 29.1667 & 4.4257 & 41.671 & 33.2633 & 0.72944 \\
\hline Blurring & 29.1667 & 0.46727 & 51.4351 & 16.8768 & 0.91365 \\
\hline Sharpening & 29.1667 & 2.585 & 44.0062 & 46.2185 & 0.91576 \\
\hline $\begin{array}{l}\text { Rotation } \\
\text { by 90 }\end{array}$ & 29.1667 & 28.5282 & 33.5781 & 41.8768 & 0.58847 \\
\hline
\end{tabular}

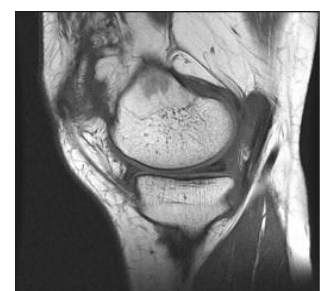

Image 3

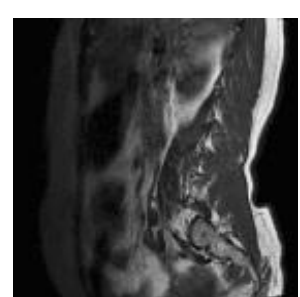

Image 4
Table 3 DWT-CDCS Result on Image 3

\begin{tabular}{|c|c|c|c|c|c|}
\hline \multicolumn{6}{|c|}{ DWT-CDCS Results after various attacks on Image 3 } \\
\hline Attacks & $\begin{array}{c}\text { PBS } \\
(\%)\end{array}$ & MSE & $\begin{array}{c}\text { PSNR } \\
(\mathbf{d B})\end{array}$ & $\begin{array}{c}\text { BER } \\
(\%)\end{array}$ & $\begin{array}{c}\text { NCC of } \\
\text { ROI }\end{array}$ \\
\hline $\begin{array}{c}\text { Without } \\
\text { Attack }\end{array}$ & $\begin{array}{c}29.166 \\
7\end{array}$ & $\begin{array}{c}0.3064 \\
7\end{array}$ & 53.267 & 0 & 0.9629 \\
\hline $\begin{array}{c}\text { Salt \& } \\
\text { Paper }\end{array}$ & $\begin{array}{c}29.166 \\
7\end{array}$ & $\begin{array}{c}0.5676 \\
5\end{array}$ & 50.59 & 1.1905 & 0.95872 \\
\hline Gaussian & $\begin{array}{c}29.166 \\
7\end{array}$ & $\begin{array}{c}19.722 \\
3\end{array}$ & $\begin{array}{c}35.181 \\
2\end{array}$ & $\begin{array}{c}50.140 \\
1\end{array}$ & 0.84461 \\
\hline Speckle & $\begin{array}{c}29.166 \\
7\end{array}$ & $\begin{array}{c}19.237 \\
4\end{array}$ & $\begin{array}{c}35.289 \\
3\end{array}$ & $\begin{array}{c}51.050 \\
4\end{array}$ & 0.84864 \\
\hline Poisson & $\begin{array}{c}29.166 \\
7\end{array}$ & $\begin{array}{c}8.1625 \\
39.012 \\
6\end{array}$ & $\begin{array}{c}48.529 \\
4\end{array}$ & 0.94746 \\
\hline
\end{tabular}




\begin{tabular}{|c|c|c|c|c|c|} 
Blurring & $\begin{array}{c}29.166 \\
7\end{array}$ & 1.2902 & $\begin{array}{c}47.024 \\
2\end{array}$ & $\begin{array}{c}18.207 \\
3\end{array}$ & 0.96074 \\
\hline $\begin{array}{c}\text { Sharpenin } \\
\mathrm{g}\end{array}$ & $\begin{array}{c}29.166 \\
7\end{array}$ & 6.7372 & 39.846 & $\begin{array}{c}50.910 \\
4\end{array}$ & 0.95393 \\
\hline $\begin{array}{c}\text { Rotation } \\
\text { by 90 }\end{array}$ & $\begin{array}{c}29.166 \\
7\end{array}$ & $\begin{array}{c}75.101 \\
7\end{array}$ & $\begin{array}{c}29.374 \\
3\end{array}$ & $\begin{array}{c}46.008 \\
4\end{array}$ & $\begin{array}{c}0.00470 \\
1\end{array}$ \\
\hline
\end{tabular}

Table 4 DWT-CDCS Result on Image 4

\begin{tabular}{|l|c|c|c|c|c|}
\hline \multicolumn{5}{|c|}{ DWT-CDCS Results after various attacks on Image 4 } \\
\hline Attacks & $\begin{array}{c}\text { PBS } \\
(\%)\end{array}$ & MSE & $\begin{array}{c}\text { PSNR } \\
(\mathbf{d B})\end{array}$ & $\begin{array}{c}\text { BER } \\
(\%)\end{array}$ & $\begin{array}{c}\text { NCC of } \\
\text { ROI }\end{array}$ \\
\hline $\begin{array}{l}\text { Without } \\
\text { Attack }\end{array}$ & 29.1667 & 0.29618 & 53.4152 & 0 & 0.96543 \\
\hline $\begin{array}{l}\text { Salt \& } \\
\text { Paper }\end{array}$ & 29.1667 & 0.55467 & 50.6905 & 1.4006 & 0.95206 \\
\hline Gaussian & 29.1667 & 18.0405 & 35.5683 & 50.4202 & 0.80208 \\
\hline Speckle & 29.1667 & 9.2714 & 38.4594 & 43.1373 & 0.88406 \\
\hline Poisson & 29.1667 & 5.2915 & 40.895 & 42.9272 & 0.98225 \\
\hline Blurring & 29.1667 & 0.60193 & 50.3354 & 12.1849 & 0.88867 \\
\hline Sharpening & 29.1667 & 3.2694 & 42.9861 & 47.1289 & 0.96511 \\
\hline $\begin{array}{l}\text { Rotation } \\
\text { by 90 }\end{array}$ & 29.1667 & 53.3449 & 30.8599 & 47.8992 & 0.07639 \\
\hline
\end{tabular}

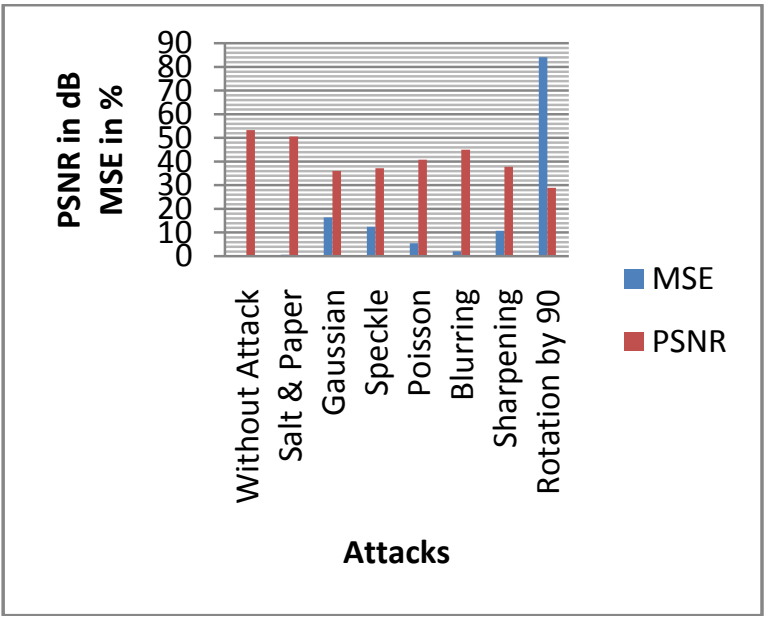

Figure 5 Graphical Chart for PSNR \& MSE

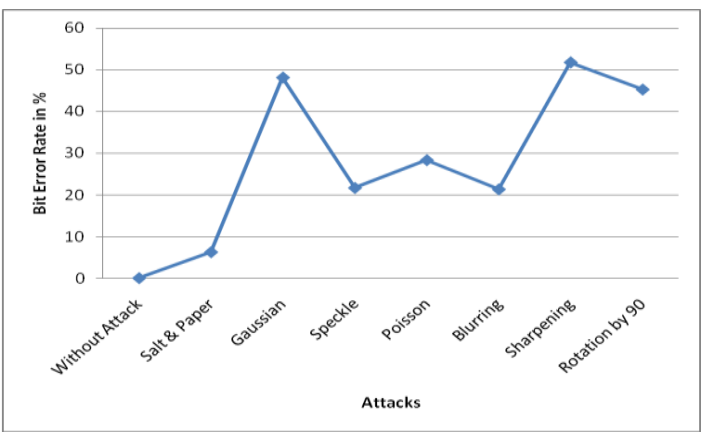

Figure 6 Graphical Chart for BER

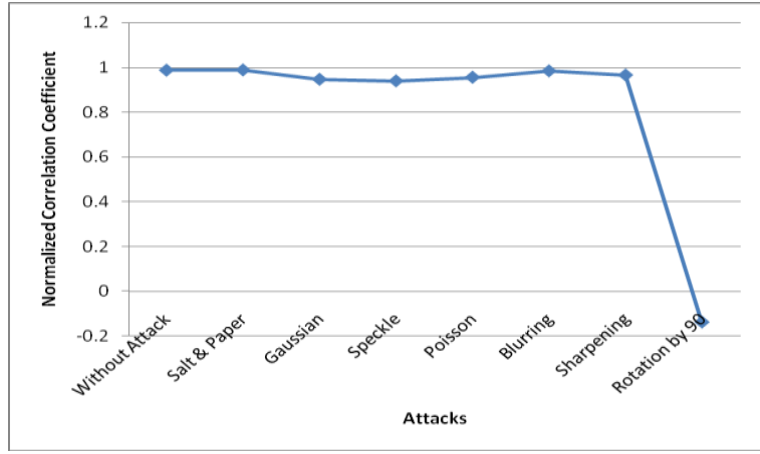

Figure 7 Graphical representation of NCC

From table 6.1 to 6.4 , it is clear that percentage of bit per saving is more than $25 \%$ so high capacity is achieved with CDCS.

Then the different watermarked images was tested for Gaussian noise, salt \& pepper noise, speckle noise, Poisson noise, blurring, sharpening, rotation attacks with same report. The PSNR obtained with the proposed algorithm is more than $54 \mathrm{~dB}$ to ensure that the watermarked image closely resembles the input medical image. The acceptable PSNR values are above $40 \mathrm{~dB}$.

It specifies data hiding is effectively done.

$\mathrm{NCC}$ is the parameter to assess the correlation between the extracted ROI and the embedded ROI. Achieved a NCC value is 0.98834 which is approximated to 1 implies that extracted ROI and the embedded ROI are same in case of without attack.

Bit error rate is the parameter to measure the number of bits gets distorted at the time of extraction which will change the data. The Bit error rate value is 0 in case of without attack.

\section{CONCLUSION}

Class dependent coding scheme is useful to save number of bits hiding capacity of data.

DWT Watermarking technique with CDCS are successfully implemented and tested for various attacks. High value PSNR is estimated. Zero bit rate error \& approximate correlation is obtained. High capacity and effective medical image data hiding is achieved using CDCS \& DWT.

\section{ACKNOWLEDGMENT}

We wish to express our warm thanks to Prof. A. D. Badadapure, HOD of Electronics \& telecommunication Department, ICOER, Pune for his support. Also we would like to thank to Dr. Adamane, Principal, ICOER, Pune for his support.

\section{REFERENCES}

[1] Sunita V. Dhavale1, and Suresh N. Mali, "High Capacity Robust Medical Image Data Hiding using CDCS with Integrity Checking", International J. of Recent Trends in Engineering and Technology, vol. 3, no. 2, May 2010

[2] Rodriguez-Colin Raul, Feregrino-Uribe Claudia, Trinidad-Blas Gershom de J. "Data Hiding Scheme for Medical Images" IEEE $7^{\text {th }}$ International Conference on Electronics, Communication and Computers,0-76952799-X/07,2007

[3] Aggeliki Giakoumaki,, Sotiris Pavlopoulos, and Dimitris Koutsouris, “ Multiple Image Watermarking Applied To 
Health Information Management" IEEE Transaction on information technology on Biomedicine, vol. 10, no. 4, pp. 722-732, Oct 2006.

[4] K. Solanki, N. Jacobsen, U. Madhow, B. S .Manjunath and S. Chandrashekhar, " Robust Image-Adaptive Data hiding using Erasure and Error Correction," IEEE Transactions on image processing, vol. 13,(2004)16271639.

[5] R.O.EI Safy, H. H. Zayed, A.EI Dessouki, "An Adaptive Steganographic Technique Based on Integer Wavelet Transform" IEEE Transaction on Image Processing vol. 978, no. 1, pp. 3778-4244-8/9, 2009.

[6] K. A. Navas, S. A. Thampy, and M. Sasikumar, "EPR Hiding in medical images for telemedicine," International Journal of Biomedical Sciences vol. 3.1 (2008) 44- 47

[7] Min. Wu , "Joint Security and Robustness Enhancement for Quantization Embedding”, IEEE Transactions On Circuit And Systems For Video Technology vol. 13,no.8 August 2003

[8] Deepthi Anand", U.C. Niranjan, "Watermarking Medical Images with Patient Information", Proceedings of the 20th Annual International Conference of the IEEE Engineering in Medicine and Biology Society, vol. 20, no. 2,1998

[9] Kurato Maeno, Qibin Sun, Shih-Fu Chang,Masayuki Suto, "New Semi-Fragile Image Authentication Watermarking Techniques Using Random Bias And Nonuniform Quantization" IEEE Transactions on Multimedia, vol. 8, no. 1, February 2006

[10] Neil F. Johnson Sushil Jajodia, "Exploring Steganography: Seeing the Unseen', IEEE Computer, vol.31, Issue 2, 1998

[11] Yiwei Wang, John F. Doherty, Robert E. Van Dyck, “A Wavelet-Based Watermarking Algorithm for Ownership Verification of Digital Images", IEEE Transactions on Image Processing, vol. 11, no. 2, February 2002

[12] Preeti Aggarwal, Renu Vig, Sonali Bhadoria, and C.G.Dethe , "Role of Segmentation in Medical Imaging: A Comparative Study", International Journal of Computer Applications (0975 8887), vol. 29 no.1, September 2011

[13] Gonzalo Alvarez1, Shujun Li and Luis Hernandez,"Analysis of security problems in a medical image encryption system," Computers in Biology and Medicine, vol. 37 (2007) 424-427.

[14] Gurpreet Kaur, Kamaljeet Kaur "Image Watermarking Using LSB (Least Significant Bit)", in International
Journal of Advanced Research in Computer Science and Software Engineering vol. 3, Issue 4, April 2013.

[15] Jasni Zain and Malcolm Clarke, "Security in Telemedicine: Issues in Watermarking Medical Images", 3rd International Conference: Sciences of Electronic, Technologies of Information and Telecommunications March 27-31, 2005

[16] B. Planitz and A. Maeder, "Medical Image Watermarking: A Study on Image Degradation", Australian Pattern Recognition Society Workshop on Digital Image Computing, WDIC 2005, Brisbane, Australia 2005

[17] Pouria Mortazavian, Mohammad Jahangiri And Emad Fatemizadeh, "A Low-Degradation Steganography Model For Data Hiding In Medical Images', Proc. $4^{\text {th }}$ IASTED Intenational Conference Visualization, Imaging And Image Processing Sep 2004

[18] Gaurav Chawla, Ravi Saini, Kamaldeep, Rajkuma Yadav, "Classification of Watermarking Based upon Various Parameters", International Journal of Computer Applications \& Information Technology vol. I, Issue II, September 2012

[19] Aayushi Verma, Rajshree Nolkha, Aishwarya Singh and Garima Jaiswal, "Implementation of Image Steganography Using 2-Level DWT Technique", International Journal of Computer Science and Business Informatics, vol.1, no.1 May 2013

[20] M. Fallahpour and M. H. Sedaaghi, "High capacity lossless Data hiding based on histogram modification", IEICE Electron. Express, vol. 4, no. 7, 2007

[21] Mahmoud El-Gayyar, "Watermarking Techniques Spatial Domain Digital Rights Seminar", in Media Informatics, University of Bonn, Germany, May 2006

[22] S. Voloshynovskiy, S. Pereira, T. Pun, J.J. Eggers and J.K. Su, "Attacks on Digital Watermarks: Classification, Estimation-based Attacks and Benchmarks", IEEE Communication Magazine, August 2001

[23] A White paper on "Digital Watermarking: A Technology Overview", Wipro Technologies, pp. 2 -8. Aug. 2003.

[24] W.R. Hendee, "Medical Imaging Physics" 2002

[25] Syed Ali Khayam, "The Discrete Cosine Transform (DCT): Theory and Application", Michigan State University March 2003 